\title{
IMMUNOCYTOCHEMICAL LOCALIZATION OF GLUTAMIC ACID DECARBOXYLASE IN NORMAL AND DEAFFERENTED SUPERIOR COLLICULUS: EVIDENCE FOR REORGANIZATION OF $\gamma$-AMINOBUTYRIC ACID SYNAPSES ${ }^{1}$
}

\author{
CAROLYN R. HOUSER, ${ }^{2}$ MARIKO LEE, AND JAMES E. VAUGHN \\ Division of Neurosciences, City of Hope Research Institute, Duarte, California 91010
}

Received January 24, 1983; Revised April 14, 1983; Accepted May 5, 1983

\begin{abstract}
There is circumstantial evidence that GABAergic synaptic terminals in the superior colliculus might become reorganized in response to a loss of the retinal innervation of this brain region. The present investigation tests this possibility by identifying $\gamma$-aminobutyric acid (GABA) neurons and their synaptic relationships with an immunocytochemical localization of the GABA-synthesizing enzyme, glutamic acid decarboxylase (GAD), and by comparing these synaptic relationships in normal superior colliculus with those present 6 and 16 weeks after unilateral eye removal. In normal superior colliculus, light microscopy revealed a much denser concentration of GAD-positive reaction product in the superficial layers than in the intermediate and deep collicular layers. Most of this reaction product was contained within small, punctate structures, but GAD-positive somata and proximal dendrites also were observed. Electron microscopy showed that GAD was localized in numerous synaptic terminals, including those that were the presynaptic elements of dendrodendritic synapses. The vast majority of GAD-positive presynaptic elements formed symmetric synaptic junctions. In addition, GAD-positive profiles frequently were postsynaptic to unstained retinal terminals as well as presynaptic to unstained dendritic profiles and thus participated in serial synaptic relationships. Furthermore, both retinal and GAD-positive elements commonly were presynaptic to the same postsynaptic dendrite, and often these synaptic contacts were adjacent to each other. In deafferented specimens, profiles with the characteristics of retinal axon terminals were not observed, whereas there appeared to be no reduction of GAD-positive synaptic profiles. However, there was a marked and statistically significant increase in the proportion of GADpositive presynaptic terminals that formed asymmetric synaptic contacts in superior colliculus deprived of retinal input. This change indicates that partial deafferentation induces a reorganization of GAD-positive synapses. The long-term presence of the GABA-synthesizing enzyme within reorganized synaptic terminals also suggests that such presynaptic elements could produce and, presumably, release neurotransmitter.
\end{abstract}

Previous investigations have sought evidence in a number of different brain regions for lesion-induced synaptic reorganization of neurons that use $\gamma$-aminobutyric acid (GABA) as a neurotransmitter, but such alterations still remain to be demonstrated unequivocally. An early

\footnotetext{
${ }^{1}$ This work was supported by United States Public Health Service Grant NS18858 from the National Institute of Neurological and Communicative Disorders and Stroke. We gratefully acknowledge Drs. E. Roberts, K. Saito, and J. -Y. Wu for preparing and characterizing the anti-GAD serum used in this research. We also wish to thank Lynn Anderson, Robert P. Barber, Colleen Heublein, Dr. Dee Ann Matthews, Christine Vaughn, and Dr. Cynthia C. Wimer for their help with various aspects of this study.

${ }^{2}$ To whom correspondence should be addressed.
}

suggestion that GABA neurons might be capable of plastic changes came from Nadler and colleagues' (1974) biochemical analysis of the GABA-synthesizing enzyme glutamic acid decarboxylase (GAD) in rat hippocampal formation following lesions of the entorhinal cortex. Although these investigators reported a chronic increase in GAD activity in the outer molecular layer of the partially deafferented dentate gyrus, it was not possible to determine from their results whether this increase was due to: (1) increased numbers of GABAergic axon terminals, (2) changes in the biochemical characteristics of GAD within existing terminals, (3) sampling variations, or (4) postlesion changes in tissue volume (Nadler et al., 1974; Storm-Mathisen, 1976, 1977). Indeed, based on 
subsequent investigations, Nadler and co-workers (Nadler et al., 1977; Nadler, 1981) have suggested that the plasticity of the GABA system in dentate gyrus may be due to factors other than a morphological reorganization of GABAergic axon terminals. Nevertheless other investigators, using essentially the same experimental paradigm as Nadler et al. (1974), recently have claimed a demonstration of a rearrangement of GABAergic fibers in GAD-immunocytochemical preparations of adult rat dentate gyrus (Goldowitz et al., 1982). However, electron microscopic evidence supporting the proposed morphological changes has not yet been presented. Furthermore, the observed changes in GAD-positive patterns could have resulted from tissue shrinkage, and this potential artifact is extremely difficult to exclude satisfactorily.

Because tissue shrinkage presents a formidable problem to studies of this kind, we have chosen an approach whereby the results are unlikely to be clouded by lesioninduced decreases in tissue volume, and whereby possible plastic changes of GABA neurons can be examined at the ultrastructural level. An appropriate system for this approach is provided by the superficial layers of rat superior colliculus. The retinal projection to this brain region is known to terminate in boutons that contain spheroidal synaptic vesicles ( $\mathrm{S}$ terminals) and form asymmetric synaptic junctions (Lund, 1969; Sterling, 1971; Valverde, 1973). In rats unilaterally enucleated as adults, it has been found that the number of such axon terminals is dramatically and chronically reduced in the contralateral superior colliculus (Lund and Lund, 1971a, b). As a result, the majority of synaptic terminals remaining within the superficial layers of the deafferented superior colliculus are those characterized by flattened or pleomorphic vesicles ( $\mathrm{F}$ terminals). Moreover, these $\mathrm{F}$ terminals are found to form asymmetric synaptic contacts much more frequently than they do in normal or control specimens. Lund and Lund (1971a, b) have interpreted their observations as meaning that some $F$ terminals become reorganized to occupy the asymmetric synaptic sites that are vacated by degenerated retinal afferents. In contrast to the studies described above, the Lunds' results involve a change in the ratio of $\mathrm{F}$ terminals that form symmetric and asymmetric synapses, and it is very difficult to envisage how this could be affected by postlesion decreases in tissue volume.

There are several reasons for suspecting that at least some of the F terminals described by Lund and Lund (1971a, b) might be GABAergic. First, the superficial layers of the superior colliculus are known to contain high concentrations of GAD-positive immunoreactivity (Ribak et al., 1980), and biochemical studies show that this collicular region exhibits high values for both $\mathrm{GAD}$ activity and GABA levels that are not dependent on the integrity of the major extrinsic afferent pathways (Okada, 1976). Second, GAD-positive synaptic terminals in numerous other brain regions (Wood et al., 1976; Oertel et al., 1981; Ribak et al., 1981) predominantly display morphological characteristics that are very similar to those of the F terminals in the superior colliculus. Thus, there is circumstantial evidence supporting the possibility that GABAergic terminals in the superior colliculus might become reorganized in response to a loss of retinal innervation. The present investigation tests this possibility by comparing the synaptic relationships of GAD-positive terminals in normal superior colliculus with those occurring in this brain region following experimental destruction of the retinal input. A preliminary report of our findings has been presented previously (Houser and Vaughn, 1981).

\section{Materials and Methods}

\section{Animals}

A total of 10 adult Sprague-Dawley rats were used in this investigation. Four of these subjects were nonenucleated rats, two of which received cerebroventricular injections of colchicine $48 \mathrm{hr}$ before sacrifice by a procedure described previously (Houser et al., 1980). This treatment was performed to enhance GAD-positive product within cell bodies and dendrites (Ribak et al., 1978), and both of the colchicine-treated rats were used exclusively for light microscopic preparations. The other two normal rats provided material for electron microscopy. The six experimental rats each had one eye surgically removed following Nembutal anesthesia. These rats were enucleated at 7 weeks of age, and they weighed 225 to $250 \mathrm{gm}$ at the time of surgery. Three experimental rats were sacrificed 6 weeks after eye removal, while the other three had a postoperative survival period of 16 weeks. All of the enucleated rats were males, whereas both males and females comprised the nonenucleated sample. Controls for the effects of eye removal were provided by the superior colliculi of normal, unoperated rats and by the ipsilateral superior colliculi of enucleated rats.

\section{Tissue preparation}

Rats were anesthetized by intraperitoneal injections of Nembutal and, for light microscopy, they were perfused through the heart with a solution of $4 \%$ paraformaldehyde in $0.12 \mathrm{M}$ phosphate buffer, $\mathrm{pH} 7.2$ to 7.4 . Small amounts of glutaraldehyde $(0.1 \%)$ and $\mathrm{CaCl}_{2}$ $(0.002 \%)$ were added to the paraformaldehyde fixative to perfuse rats for electron microscopic examination. For both light and electron microscopic preparations, the brains remained in situ for about $2 \mathrm{hr}$ at room temperature, and then the midbrain regions of the brainstems were removed and stored overnight at $4^{\circ} \mathrm{C}$ in the paraformaldehyde fixative without glutaraldehyde. Specimens for light microscopy were rinsed in phosphate buffer and infiltrated with a cryoprotectant solution of $30 \%$ sucrose. Transverse sections of the superior colliculi were cut at a thickness of $40 \mu \mathrm{m}$ on a cryostat following freezing of the tissue blocks with dry ice. Control and experimental (enucleated) specimens for electron microscopy were trimmed to include the region of the midbrain dorsal to the cerebral aqueduct containing the superior colliculi, and the deafferented sides of experimental specimens were marked for subsequent identification. Transverse sections, $100 \mu \mathrm{m}$ thick, were made on a Vibratome without prior cryoprotection or freezing. Sections from the central one-third of the rostral-caudal extent of the superior colliculus were selected and, after immunocytochemical incubations (see below), specimens for electron microscopy were obtained from the middle 
one-third of the medial-lateral extent of control and deafferented superior colliculi.

\section{Immunocytochemistry}

Antiserum. The antiserum used in this study was produced in rabbit against purified GAD from mouse brain synaptosomal preparations (Wu et al., 1973). This antiserum has been characterized by detailed immunological studies (Saito et al., 1974), and its specificity in immunocytochemical investigations has been demonstrated in numerous appropriate test regions of the CNS (Vaughn et al., 1981a review).

Immunocytochemical incubations. Free-floating sections were processed with a modification of the unlabeled peroxidase-antiperoxidase (PAP) method (Sternberger, 1979; Vaughn et al., 1981a) that employs the Fab fragments of PAP IgG molecules (Slemmon et al., 1980). The tissue sections were preincubated in normal rat serum to mask nonspecific adsorption sites and then rinsed in phosphate-buffered saline (PBS; $75 \mathrm{~mm}$ sodium phosphate and $75 \mathrm{~mm}$ sodium chloride). Next, the specimens were incubated for $2 \mathrm{hr}$ in either anti-GAD rabbit serum or nonimmune (control) rabbit serum. The dilutions of both anti-GAD and control sera were $\times 50$. Following incubation in primary antiserum or control serum, specimens were rinsed in PBS, incubated in goat anti-rabbit IgG serum, rinsed, and incubated in rabbit PAP-Fab complex as described in detail previously (Vaughn et al., 1981a). The sections were then reacted with $3,3^{\prime}$-diaminobenzidine tetrahydrochloride $(30 \mathrm{mg} / 50 \mathrm{ml}$ of PBS) and $0.006 \% \mathrm{H}_{2} \mathrm{O}_{2}$. Sections containing both control (ipsilateral) and deafferented colliculi were processed through the immunocytochemical reagents before they were trimmed for electron microscopy (see above). This ensured that experimental and control tissue was subjected to identical immunocytochemical conditions. Specimens for light microscopy were treated for $30 \mathrm{sec}$ in $0.1 \%$ osmium tetroxide, whereas those for electron microscopy were treated for $1 \mathrm{hr}$ in $2 \%$ osmium tetroxide. Standard procedures were used to mount light microscopic sections and to prepare specimens for electron microscopic examination.

\section{Quantitative electron microscopic analysis}

Samples for this analysis were obtained from the control (ipsilateral) and deafferented superior colliculi of enucleated rats. A well trained electron microscopist, who was not informed of the purpose of this research, randomly photographed nonoverlapping fields of the superficial layers of the superior colliculus that contained GAD-positive terminals. The ultrathin sections were coded to prevent the electron microscopist from knowing which were obtained from experimental or control specimens. Negatives were taken at a primary magnification of $\times 11,400$ and electron micrographs were printed at a final magnification of $\times 28,600$. Twenty to 30 such electron micrographs were obtained for each control and deafferented superior colliculus included in this study. These micrographs yielded 35 to 57 classifiable (see below) synaptic junctions formed by GAD-positive terminals from each control and experimental specimen, and the totals of GAD-positive synapses evaluated in this study were 258 from control specimens and 286 from deafferented specimens.

All electron micrographs were coded so that GADpositive synapses were classified and enumerated without knowledge of whether they were derived from control or deafferented material. All GAD-positive synapses were classified independently by two of the investigators on the bases of previous descriptions of synaptic junctions in rat superior colliculus (Lund, 1969; Lund and Lund, $1971 \mathrm{a}, \mathrm{b})$ and information obtained in the present study. GAD-positive terminals were classified as forming: (1) asymmetric synapses, if the postsynaptic density (PSD) was greater than approximately $30 \mathrm{~nm}$ in thickness; (2) symmetric synapses, if the postsynaptic specialization was approximately $17 \mathrm{~nm}$ or less in thickness; (3) intermediate synapses, if the PSD was neither clearly asymmetric nor symmetric; and (4) as combination synapses, if the PSD exhibited adjacent regions clearly characteristic of both asymmetric and symmetric contacts. The results from the two independent analyses were in good agreement. The few cases of differential classification were discussed and were reclassified if a consensus could be reached, or rejected from the sample if there was no clear agreement. Only an insignificant percentage $(0.005 \%)$ of the total synaptic sample was eliminated for this reason. The results of this study were analyzed statistically by $t$ tests.

\section{Results}

\section{Normal superior colliculus}

GAD-positive elements. Light microscopy showed a much denser concentration of GAD-positive reaction product in the superficial layers of the superior colliculus than in the intermediate and deep layers (Figs. 1 to 3 ). Most of this reaction product was contained within punctate structures resembling terminals or transversely sectioned profiles of neuronal processes (Figs. 5 to 8 ). In addition, GAD-positive cell bodies were present in the superficial layers, and short GAD-positive, dendrite-like processes emanated from some of these stained somata (Figs. 3, and 6 to 8 ). No specific immunoreactivity was observed in specimens incubated in control serum (Fig. 4).

Electron microscopic examination of the superficial collicular layers demonstrated the presence of many GAD-positive presynaptic terminals, and the vast majority of these terminals formed symmetric synaptic contacts (Figs. 9 and 10). Although GAD-positive reaction product partially obscured the morphology of the synaptic vesicles within these terminals, some of the vesicles were pleomorphic in shape. GAD-positive reaction product also was observed in profiles that displayed characteristics of dendrites; namely, numerous longitudinally oriented microtubules and, in larger profiles, occasional cisternae of the granular endoplasmic reticulum and Golgi apparatus (Figs. 11 and 12). In addition, these processes were the presynaptic (Fig. 12), as well as the postsynaptic, components of synaptic junctions, and they therefore represented some of the presynaptic dendrites known to be present in the superior colliculus (Lund, 1969; Sterling, 1971; Valverde, 1973; Tigges and Tigges, 

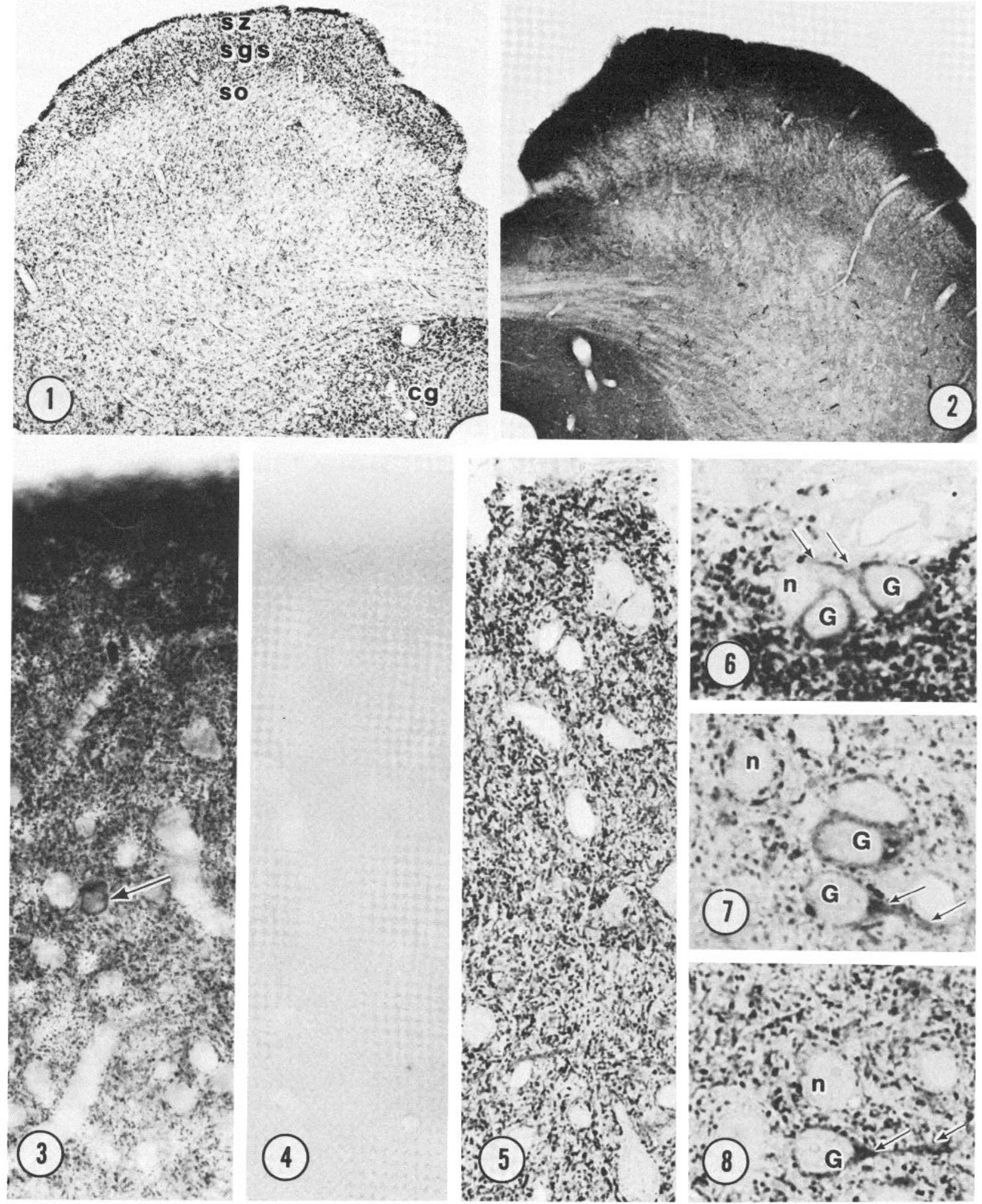

Figures 1 to 8 . Light micrographs of normal superior colliculus. Figures 1 to 4 are 40 - $\mu$ m-thick frozen sections, while Figures 5 to 8 are 1 - to $2-\mu \mathrm{m}$-thick, plastic embedded sections. Figure 1 is a Nissl-stained section, included for orientation ( $s z$, stratum zonale; sgs, stratum griseum superficiale; so, stratum opticum; cg, central gray). Magnification $\times 44$. Figure 2 is an adjacent section incubated in anti-GAD serum showing an intense band of staining approximately $250 \mu \mathrm{m}$ thick in sz and sgs (Magnification $\times 36$ ). Figure 3 shows the GAD-positive staining of the superficial superior colliculus at higher magnification, and a GADpositive soma is designated by an arrow (Magnification $\times 540$ ). Figure 4 illustrates a lack of staining in a section of superficial superior colliculus incubated in control serum (Magnification $\times 510$ ). Figure 5 shows that the intense GAD-positive staining of superficial collicular layers mainly is due to numerous punctate structures (Magnification $\times 560)$. GAD-positive somata $(G)$ are shown in Figures 6 to 8 , and some of these cell bodies give rise to GAD-positive processes (arrows). GAD-positive punctate structures are adjacent to both GAD-positive $(G)$ and GAD-negative $(n)$ somata (Magnification: Fig. $6, \times 1000 ;$ Fig. $7, \times 850$; Fig. $8, \times 850)$. 

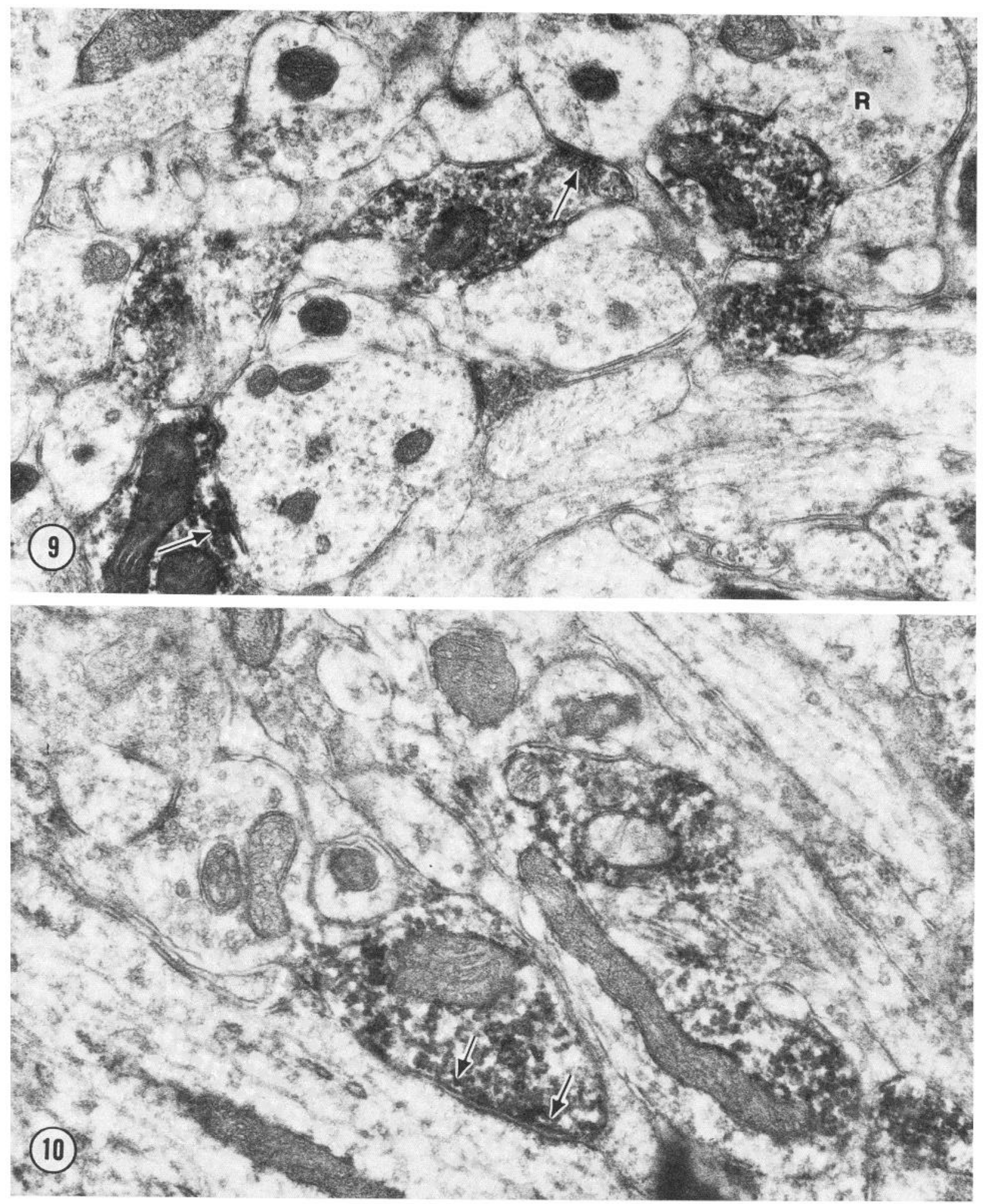

Figures 9 and 10. Electron micrographs from the superficial layers of normal superior colliculus incubated in anti-GAD serum. In Figure 9, two GAD-positive terminals form symmetric synaptic contacts (arrows) with GAD-negative dendritic profiles. Part of a GAD-negative retinal terminal $(R)$ is located in the upper right corner (Magnification $\times 31,000$ ). A higher magnification of a symmetric synapse (arrows) between a GAD-positive terminal and a GAD-negative dendrite is shown in Figure 10 (Magnification $\times 38,700$ ). In these and all subsequent figures, arrows point from presynaptic elements toward their postsynaptic targets.

1975). Synaptic contacts formed by these presynaptic dendrites usually were symmetric (Fig. 12). The reaction product in GAD-positive dendrites often was associated with microtubules and also was concentrated around clusters of vesicles located at potential presynaptic sites (Figs. 11 and 12). However, in some cases, the main shafts of GAD-positive dendrites contained only sparse reaction product, whereas synaptic vesicle-filled gem- 

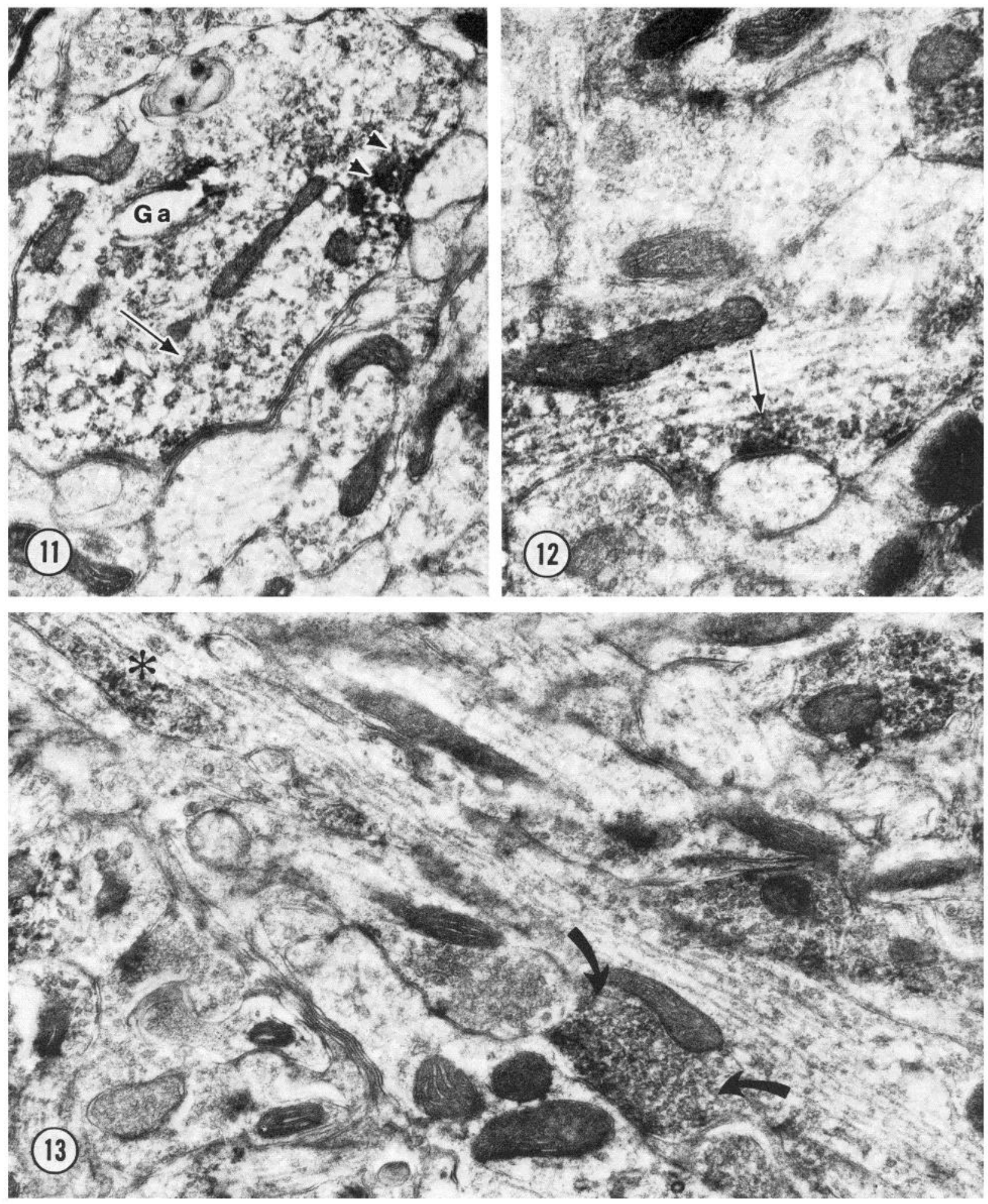

Figures 11 to 13. Electron micrographs of GAD-positive dendrites in normal superior colliculus. Figure 11 shows a large profile of a GAD-positive dendrite that contains cisternae and vesicles of the Golgi apparatus $(\mathrm{Ga})$, as well as what might be a grazed section of granular endoplasmic reticulum (arrow). A dense accumulation of reaction product (arrowheads) is located at a possible presynaptic site apposed to a GAD-negative profile (Magnification $\times 28,600$ ). A distal GAD-positive dendrite, lightly labeled in regions containing synaptic vesicles, is the presynaptic component of the symmetric synapse (arrow) shown in Figure 12 (Magnification $\times 37,200$ ). In Figure 13, a longitudinally sectioned dendrite displays intense GAD-positive staining associated with synaptic vesicles in the dendritic shaft $(*)$, as well as in a gemmule-like dendritic excrescence (curved arrows). However, much of the dendrite lacks reaction product (Magnification $\times 28,600$ ). 
mules protruding from these shafts were stained intensely (Fig. 13). Gemmules lacking continuity with a dendritic shaft in the plane of section examined could not have been distinguished from axon terminals. Therefore, for quantitative analysis (see below), we have designated all GAD-positive presynaptic structures simply as synaptic terminals without attempting to judge whether they arose from axons or dendrites.

Synaptic relationships. When GAD-positive terminals were presynaptic elements, they predominantly formed symmetric synaptic contacts with GAD-negative dendritic profiles (Figs. 9 and 10), although they also were observed to make symmetric synapses with neuronal somata. In circumstances where GAD-positive terminals were postsynaptic elements, they frequently were associated with GAD-negative presynaptic terminals that were characterized by round synaptic vesicles, large relatively pale mitochondrial profiles, and asymmetric synaptic junctions (Fig. 14). Previous studies have identified such terminals as belonging to retinal ganglion cells (Lund, 1969; Sterling, 1971; Valverde, 1973). Such terminals often synapsed with GAD-positive terminals that, in turn, made synaptic contacts with unstained dendritic profiles, thereby completing serial synaptic relationships (Figs. 15 and 16). Similar serial synapses among nonretinal terminals, GAD-positive terminals, and unstained dendrites were observed but were exceedingly rare in comparison to the ones involving retinal terminals. Individual retinal terminals frequently established multiple synaptic junctions, and sometimes they synapsed with unstained dendrites as well as with a GAD-positive postsynaptic element (Fig. 14). In addition, both retinal and GAD-positive terminals commonly were presynaptic to the same dendritic profile, and often the synaptic contacts were adjacent to one another (Fig. 17).

Another type of synaptic relationship observed was one where both the pre- and the postsynaptic components were GAD positive. Although this relationship appeared to be relatively rare, the GAD-positive postsynaptic elements in the observed examples were dendrites and the synaptic contacts were symmetric. Several of the GADpositive postsynaptic dendrites involved in this type of synapse exhibited presynaptic accumulations of vesicles elsewnere along their lengths and formed symmetric synapses with unstained postsynaptic dendrites at these sites, thereby completing serial synaptic relationships of GAD-positive terminals to GAD-positive presynaptic dendrites to GAD-negative postsynaptic dendrites. The synaptic organization of GAD-positive and retinal terminals in the control (ipsilateral) superior colliculus of enucleated rats was essentially the same as that just described for normal, unoperated animals.

\section{Deafferented superior colliculus}

General electron microscopic observations. No striking differences were observed among the deafferented colliculi from 6 - or 16 -week postoperative rats; therefore, the results from these two groups will be described together. The superficial layers of deafferented superior colliculus were essentially devoid of retinal terminals. However, there was no apparent reduction of GAD-positive terminals. When these structures were presynaptic elements, the majority of them formed symmetric synaptic contacts (Fig. 18), as observed in normal and control specimens. However, substantially more of these terminals formed asymmetric junctions than in control specimens (Figs. 19 to 21; see also quantitative analysis below). Both GAD-positive presynaptic dendrites and isolated terminals participated in asymmetric synapses. A relationship that appeared to be unique to deafferented specimens was the occurrence of GAD-positive terminals that formed synaptic contacts with the characteristics of both asymmetric and symmetric synapses and these junctions were termed "combination" synapses (Fig. 23). In addition, deafferented specimens contained GAD-positive synapses where the postsynaptic densities were intermediate in thickness to those of either patently asymmetric or symmetric junctions (Figs. 21 and 22).

GAD-positive structures also were the postsynaptic components of synaptic junctions in deafferented superior colliculus. The presynaptic elements of these junctions formed asymmetric contacts, had numerous spheroidal vesicles, and contained small, dark mitochondrial profiles. Furthermore, serial synaptic relationships involving these nonretinal terminals, GAD-positive terminals, and unstained dendrites were observed in deafferented specimens more frequently than in controls. Synapses among GAD-positive pre- and postsynaptic elements, similar to those observed in normal and control specimens, also were present in deafferented superior colliculus. In contrast to controls, however, some of these synapses in deafferented specimens exhibited asymmetric contacts. Naked or glial-covered asymmetric postsynaptic sites, such as those described by Lund and Lund $(1971 \mathrm{a}, \mathrm{b})$ in deafferented adult superior colliculus could not be identified conclusively in our experimental specimens.

Quantitative electron microscopic analysis. In the superficial layers of control superior colliculus, $98.4 \%$ of the synapses formed by GAD-positive presynaptic terminals were classified as symmetric, while the remaining $1.6 \%$ were judged to be asymmetric (also see Table I). No examples of GAD-positive terminals forming either intermediate or combination synaptic junctions (see above) were observed. In contrast, samples of GAD-positive synapses from deafferented specimens revealed a few of each of these two synaptic types, and they were somewhat more common in 6-week than in 16-week postoperative specimens (Table I). The salient finding of this analysis was that deafferented superior colliculus showed a statistically significant increase in GAD-positive presynaptic terminals that formed asymmetric synapses, and such junctions accounted for $11.5 \%$ of the total GADpositive synaptic sample $(t=4.94 ; p<0.01)$. GAD. positive presynaptic terminals forming symmetric synaptic junctions in deafferented specimens decreased significantly to $83.6 \%$ of the total GAD-positive synaptic sample $(t=4.31 ; p<0.01)$. These results were obtained by combining the data from the 6- and 16-week postoperative animals. This was done since there was no statistically significant difference between these two experimental groups ( $t=0.50$, not significant).

\section{Discussion}

There is abundant evidence supporting a conclusion that the GABA-synthesizing enzyme, GAD, is a defini- 

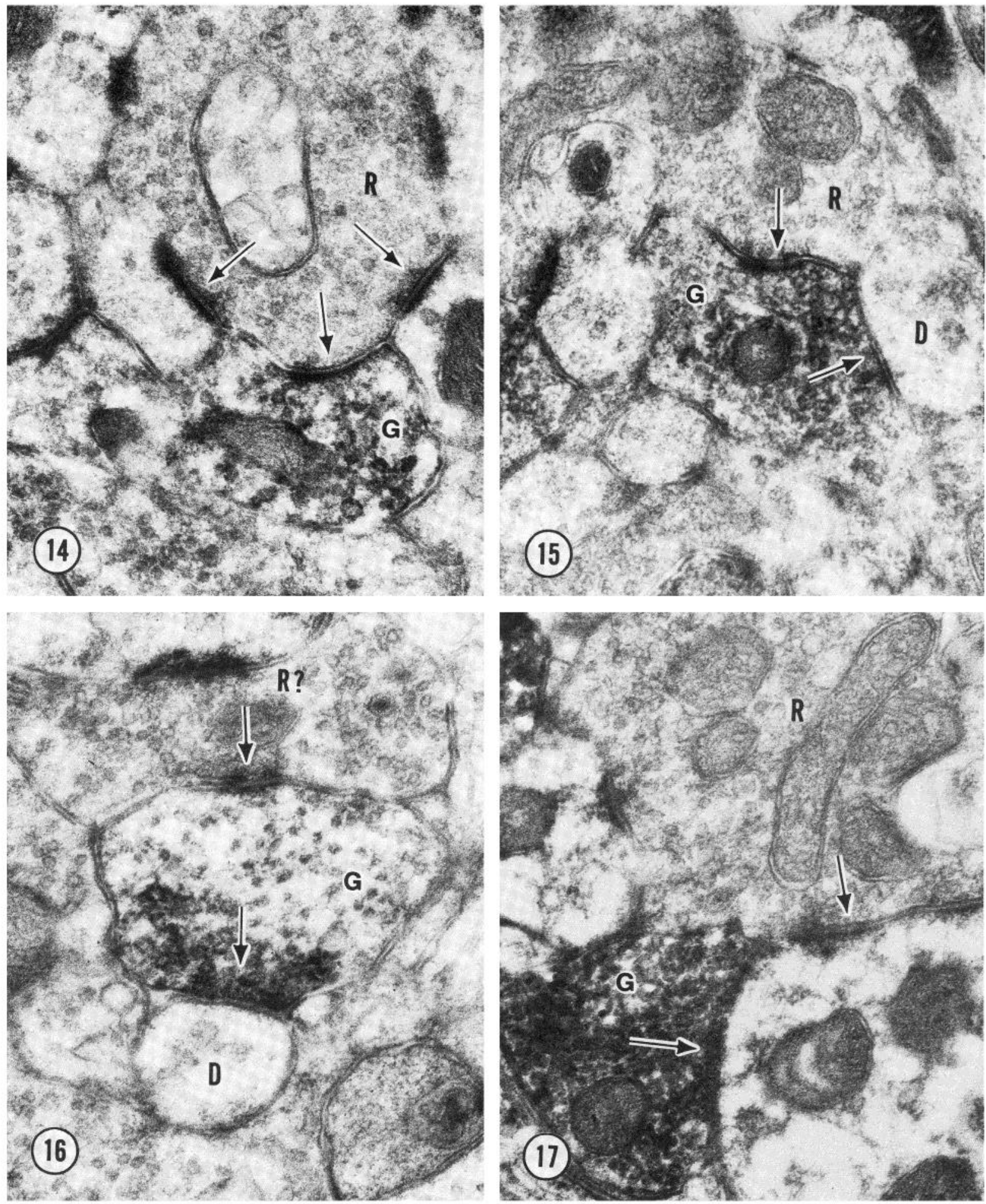

Figures 14 to 17. Electron micrographs illustrating the relationships between retinal terminals and GAD-positive profiles in normal superior colliculus. In Figure 14, a GAD-negative retinal terminal $(R)$ forms multiple asymmetric contacts (arrows) with GAD-positive $(G)$ and GAD-negative postsynaptic elements (Magnification $\times 53,600$ ). A serial synaptic complex is shown in Figure 15 where a retinal terminal $(R)$ forms an asymmetric contact (upper arrow) with a GAD-positive terminal $(G)$ that, in turn, makes a symmetric junction (lower arrow) with a GAD-negative dendritic $(D)$ profile $($ Magnification $\times 41,000)$. A similar serial synaptic relationship is illustrated in Figure 16 except, in this case, the first presynaptic element $(R$ ?) cannot be identified unequivocally as a retinal terminal and the middle GAD-positive element $(G)$ has the appearance of a transversely sectioned shaft of a presynaptic dendrite. The final element is a small GAD-negative dendritic $(D)$ profile $($ Magnification $\times 52,700)$. In Figure 17, a GAD-negative retinal terminal $(R)$ and a GAD-positive terminal $(G)$ form adjacent synaptic junctions (arrows) with the same postsynaptic dendrite (Magnification $\times 47,900$ ). 

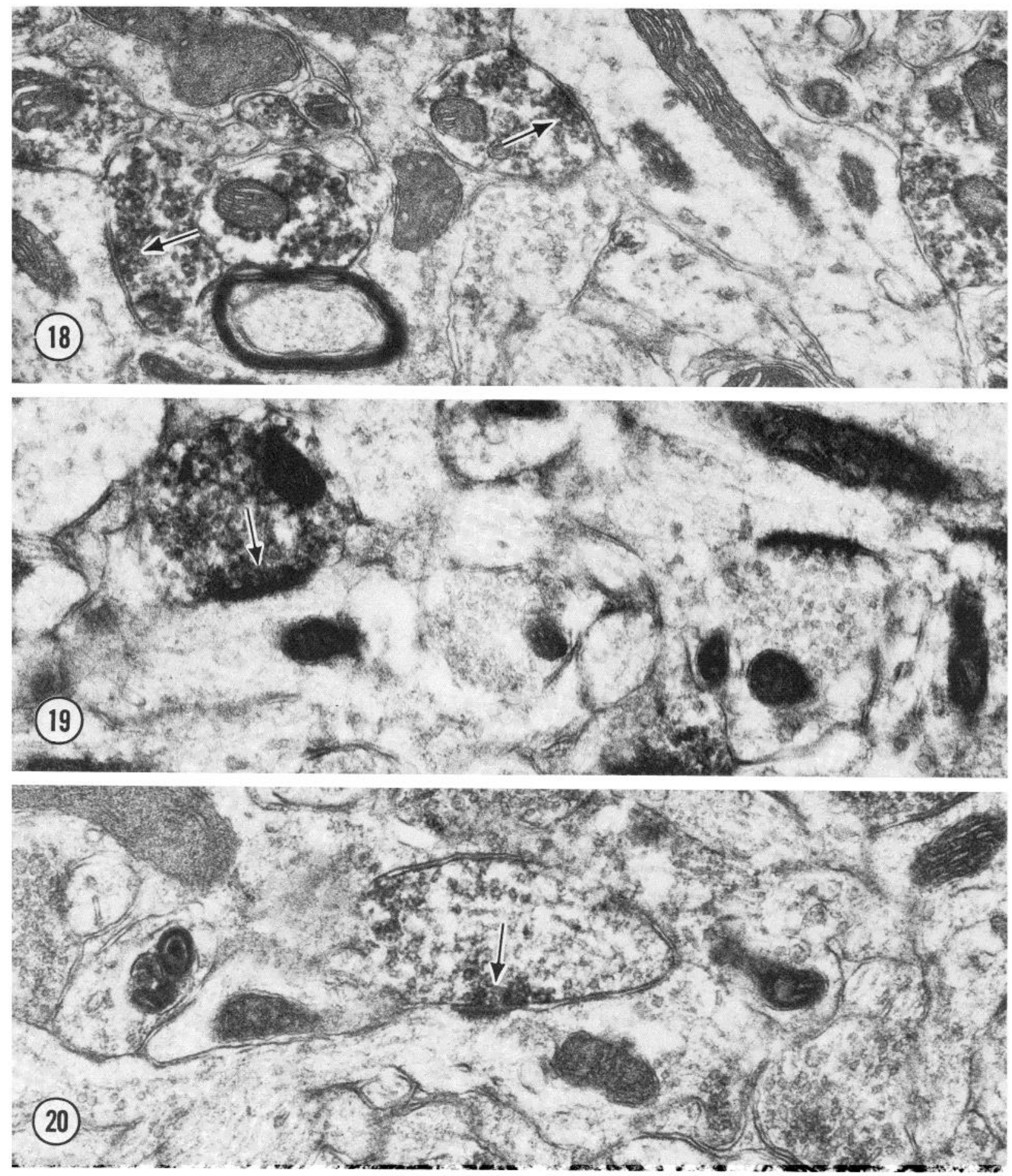

Figures 18 to 20. Electron micrographs of GAD-positive synaptic terminals in deafferented superior colliculus. As illustrated in Figure 18, the majority of GAD-positive terminals in deafferented colliculus form symmetric synapses (arrows) with GADnegative dendrites (Magnification $\times 32,200$ ). However, as shown in Figures 19 and 20, asymmetric junctions (arrows) formed by GAD-positive synaptic terminals (Fig. 19) and probable presynaptic dendrites (Fig. 20) with GAD-negative postsynaptic elements are present and are more common (see the text) than in normal or control specimens (Magnification: Fig. 19, $\times 40,200$; Fig. 20, $\times 39,000)$.

tive marker for neurons that use GABA as a neurotransmitter substance (Storm-Mathisen, 1977; Oertel et al., 1981; Vaughn et al., 1981a review). On this basis, the GAD-positive somata, dendrites, and synaptic terminals revealed by the present study are identified as belonging to GABA neurons. The large number of such elements observed in the superficial layers of the superior collicu- lus is consistent with the results of biochemical analyses of the GABA system in this brain region (Okada, 1976), as well as with the findings of physiological investigations that indicate GABA-mediated inhibitory phenomena may have an important role in information processing within the superior colliculus (Takahashi et al., 1977; Kayama et al., 1980). 

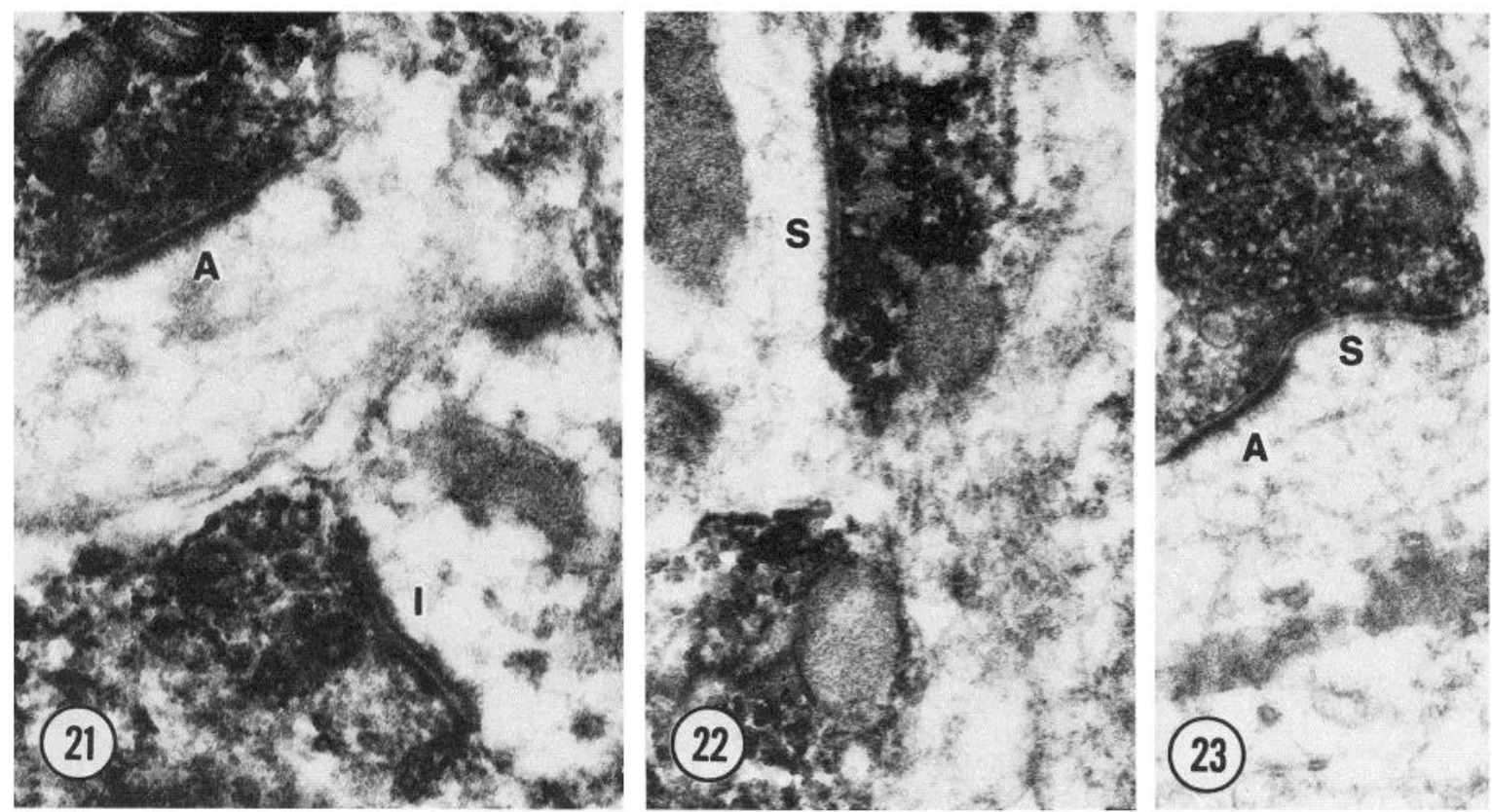

Figures 21 to 23. Electron micrographs of GAD-positive synapses in deafferented superior colliculus. Presynaptic GAD-positive elements from the same field of neuropil form asymmetric $(A)$, intermediate $(I)$, and symmetric $(S)$ synaptic contacts in Figures 21 and 22 (Magnification $\times 37,200$ ). Figure 23 illustrates a combination synapse between a GAD-positive presynaptic terminal and a GAD-negative dendrite. The left edge of this contact is asymmetric $(A)$, while its middle region is symmetric $(S)$, and the right edge displays what appears to be a punctum adhaerens (Magnification $\times 32,200$ ).

TABLE I

Types of GAD-positive synapses in samples of control and deafferented superior colliculus used for quantitative analysis

\begin{tabular}{|c|c|c|c|c|c|}
\hline \multirow{2}{*}{ Specimen } & \multirow{2}{*}{$\begin{array}{c}\text { Total No. of } \\
\text { GAD-positive } \\
\text { Synapses Classified }\end{array}$} & \multicolumn{4}{|c|}{ No. of Each Type of GAD-positive Synapse } \\
\hline & & Symmetric & Asymmetric & Intermediate & Combination \\
\hline \multicolumn{6}{|l|}{ Experimental } \\
\hline 16 weeks postoperative & $\underline{140}$ & $\underline{123}$ & $\underline{15}$ & $\underline{0}$ & $\underline{2}$ \\
\hline $\begin{array}{l}\text { Combined } 6 \text { and } 16 \text { weeks } \\
\text { postoperative }\end{array}$ & $\overline{286}$ & $\overline{239}$ & $\overline{33}$ & $\overline{7}$ & $\overline{\overline{7}}$ \\
\hline
\end{tabular}

\section{Normal superior colliculus}

\section{$G A D$-positive presynaptic dendrites}

The results of previous studies have shown that the superficial layers of the superior colliculus contain presynaptic dendrites and have suggested that these dendrites may have an inhibitory function because their presynaptic sites exhibit flattened vesicles and their synaptic contacts are symmetric (Lund, 1969; Sterling, 1971; Valverde, 1973; Tigges and Tigges, 1975). Furthermore, suggestive evidence that these presynaptic dendrites may use GABA as a neurotransmitter has been provided by radioautographic studies of tritiated GABA uptake (Streit et al., 1978; Mize et al., 1981). Results from the present study strongly indicate that some presynaptic dendrites in the superior colliculus are indeed GABAergic, and also that they exhibit plastic changes following partial deafferentation (see below).

\section{Synaptic organization}

GAD-positive presynaptic dendrites rather frequently are intercalated between retinal afferents and unlabeled postsynaptic dendrites in serial synaptic relationships.
Less commonly, they are the intermediate elements in serial synapses between nonretinal terminals and unlabeled dendrites. Even more infrequently, GAD-positive presynaptic dendrites are the interlinking components of serial synapses between GAD-positive terminals and unlabeled dendrites. Although GAD-positive presynaptic dendrites have been observed as the middle elements in all of these serial synaptic relationships, it is emphasized that GAD-positive profiles that cannot be identified precisely as dendritic also occupy this same position in these types of serial synapses. As mentioned above, it often is not possible to determine in our preparations whether such structures are the gemmule-like excrescences of presynaptic dendrites or whether they are axon terminals.

\section{Comparison with other parts of the visual pathway}

A broad pattern of synaptic organization seems to be emerging for GABA neurons in the visual system. For example, such neurons commonly have presynaptic dendrites in the retina (Wood et al., 1976; Vaughn et al., 1981b), dorsal lateral geniculate nucleus (Ogren et al., 1982; Hendrickson et al., 1983; Ohara et al., 1983), and 
the superficial layers of the superior colliculus as demonstated in the present study. In all three of these sites, the GABAergic presynaptic dendrites provide the intercalated, or middle, element in serial synaptic relationships. Furthermore, retinal afferents are the first presynaptic unit of these serial synapses in the dorsal lateral geniculate nucleus and the superior colliculus, while bipolar terminals fill this role in the retina. The final elements of these synaptic complexes are the conventional dendrites of non-GABAergic projection neurons in the retina and the dorsal lateral geniculate nucleus. Although it remains to be documented, this could also be the case in the superior colliculus since the cell bodies and dendrites of projection cells are present in the superficial superior colliculus (Graham and Casagrande, 1980; Kawamura et al., 1980). A notable difference in these complex synaptic relationships is that reciprocal synapses, such as those observed in the retina among GABAergic amacrine cell dendrites and bipolar synaptic terminals (Vaughn et al., 1981b), have not been detected in either the dorsal lateral geniculate nucleus or the superior colliculus. A striking exception to the apparent pattern of GABAergic neurons in the visual system is the visual cerebral cortex. At the present time, neither GABAergic presynaptic dendrites nor serial synapses involving GABAergic terminals have been observed in the final processing center of the visual pathway (Ribak, 1978).

\section{Sources of GAD-positive terminals}

Although the present study does not allow a precise determination of the sources of GAD-positive synaptic terminals in the superior colliculus, our results in combination with those of other investigators indicate that many of these terminals are derived from intrinsic neurons. Our observation of GAD-positive somata and dendrites in this region, as well as the fact that many of the GAD-positive synaptic profiles are parts of presynaptic dendrites, argue strongly in favor of this interpretation. Further support for this point of view comes from the results of biochemical studies where lesions of the optic tract, visual cortex, and commissural fibers of the colliculi produced no significant decrease of GAD activity in the superficial superior colliculus (Okada, 1976). Although GABAergic projections to the superior colliculus from other brain regions such as the substantia nigra have been suggested, they all appear to terminate in collicular layers deeper than those examined in the present study (Grofová et al., 1978; Vincent et al., 1978a). Thus, although there may be as yet unidentified extrinsic GABAergic projections to the superficial superior colliculus (Fonnum et al., 1979), it seems reasonable to conclude that intrinsic neurons are a major source of GABAergic terminals in this brain region.

\section{Deafferented superior colliculus}

\section{GAD-positive synapses are reorganized}

There is a marked and statistically significant increase in the proportion of GAD-positive presynaptic terminals that form asymmetric synaptic contacts in superior colliculus deprived of retinal input. This change indicates that partial deafferentation induces a reorganization of the synaptic relationships of GABA neurons. Using basically the same experimental protocol as that of the present study, Lund and Lund (1971a, b) previously have shown an increase in the percentage of $F$ terminals that occupy asymmetric synaptic sites. The results of our study and of the Lunds are remarkably similar, both qualitatively and quantitatively, and this suggests that the same phenomenon has been examined in both investigations. Our findings extend those of Lund and Lund (1971a, b) by providing firm evidence that GABA is the transmitter of synaptic terminals that undergo a postdeafferentation reorganization. Furthermore, the immunocytochemical demonstration of the GABA-synthesizing enzyme within reorganized synaptic terminals at chronic postoperative times indicates that these presynaptic elements possess the ability to produce neurotransmitter, suggesting therefore that at least the presynaptic components of the reorganized synapses are functional.

\section{Intrinsic GABA neurons participate in reorganization}

We have argued, above, that intrinsic neurons are a major source of GABA terminals in normal superior colliculus. If this is the case, it logically follows that GABA interneurons may play a major role in the synaptic reorganization that takes place in the deafferented colliculus. Furthermore, our observations that GAD-positive presynaptic dendrites are involved in postlesion synaptic changes strongly supports this conclusion. It has long been thought that intrinsic neurons might rank among the most plastic neurons in damaged or experimentally altered CNS (Rakic, 1975). The changes observed in deafferented superior colliculus provide an unequivocal example supporting this point of view.

\section{Mode(s) of reorganization}

Lund and Lund, (1971a, b) interpret their results as meaning that $F$ terminals re-occupy asymmetric sites vacated by degenerated retinal afferents. It is also possible that existing or newly sprouted terminals could form asymmetric synapses de novo rather than occupying pre-existing asymmetric sites. The results of our analyses do not provide a clear choice between these alternatives, but a discussion of the combination and intermediate synaptic junctions that we have observed exclusively in deafferented specimens may be germane to this problem. Although there were no statistically significant differences between our 6- and 16-week postoperative specimens, both combination and intermediate synapses appeared to be more common in the 6 -week animals. This hints that such synapses might have been transitional forms evolving into their chronic synaptic morphology. Perhaps the most simple explanation of combination synapses is that they represent a relatively early stage in an expansion of GABA terminals to overlay adjacent, vacated asymmetric sites. In this case, the symmetric portion of a combination contact may be the original synaptic contact, while the asymmetric part might represent the re-occupation of an adjacent, vacated site by the expanding (sprouting) GABA terminal. Intermediate synapses, on the other hand, might be most easily interpreted as de novo formation of asymmetric synapses by GABA terminals at early stages when the postsynaptic densities have not yet differentiated into their full thick- 
ness. The possibility of de novo formation of GABA synapses with asymmetric contact sites is tenable since some synapses of this type are present in normal superior colliculus, as well as in other regions of the normal CNS (McLaughlin et al., 1975; Ribak et al., 1976). Alternate interpretations of both the combination and intermediate synaptic junctions are obviously plausible. In addition, it is possible that other forms of reorganization may have occurred in the deafferented superior colliculus. For example, nonretinal afferents may fill vacated asymmetric sites, but this is difficult to determine since a decrease in tissue volume would affect the observed number of synapses formed by nonretinal terminals.

\section{Function(s) of reorganized GABA synapses}

Just as the mode of reorganization is unclear, so also is the question of whether the reorganized synapses are functional. As was mentioned above, the presence of the GABA-synthesizing enzyme in reorganized presynaptic terminals suggests that they should be capable of producing transmitter. Assuming they also can release transmitter appropriately, the next problem is whether the postsynaptic elements of reorganized synapses possess the receptors necessary for synaptic transmission. In normal superior colliculus, retinal and GABA terminals often form synapses on the same dendrite and frequently are located in close proximity to one another. This suggests that dendrites of some collicular neurons normally possess receptors for both types of synapses and that these receptors are located in approximately the same dendritic region. It is reasonable to suspect, therefore, that GABA receptors could exist at - or could easily translocate to-reorganized synaptic sites, and thereby establish a match between new pre- and postsynaptic components.

If reorganized synapses are capable of synaptic transmission, the functional significance of GABAergic and, presumably, inhibitory inputs to sites that would normally receive excitatory synapses (McIlwain and Fields, 1971 ) is not at all clear. One possiblity is that the reorganized synapses could release GABA to suppress spontaneous activity of deafferented neurons which might otherwise interfere with the normal processing of information by the undamaged colliculus. Alternatively, the functional significance of the reorganized GABA synapses may not reside in conventional information processing per se, since it is conceivable that the role of these synapses may be to provide a supplementary "nourishment" of trophic factor(s) to the deafferented neurons.

\section{Generality of GABA neuronal reorganization}

Despite the present ambiguities concerning the functional significance of reorganized GABA synapses and the precise manner in which reorganization is achieved, it is clear that such synaptic terminals are capable of a significant degree of morphological plasticity in the superficial layers of the superior colliculus. There is available, indirect evidence that this may also be the case in other brain areas. For example, following partial deafferentation, presynaptic terminals characterized by flattened or pleomorphic vesicles have been found apposed to asymmetric postsynaptic sites in diverse regions of the CNS (Westrum and Black, 1971; Gentschev and
Sotelo, 1973; Rustioni and Sotelo, 1974; Smith and Ebner, 1980). If, as is the case in the superior colliculus, some of these $F$ terminals are derived from GABA neurons, it would indicate a lesion-induced reorganization of GABA synapses. This possibility, in conjunction with suggestive biochemical and immunocytochemical evidence favoring possible plastic changes of GABA neurons in many different neural regions (Nadler et al., 1974; Emson et al., 1976; Vincent et al., 1978b; Gilad and Reis, 1979; Nieoullon and Dusticier, 1981; Goldowitz et al., 1982), suggests that a reorganization of GABA synapses and/or other types of plastic changes may be a rather common characteristic of GABA interneurons distributed throughout the brain and spinal cord.

\section{References}

Emson, P. C., A. Björklund, and U. Stenevi (1976) Possible regeneration of $\gamma$-aminobutyric acid-containing fibres into irides transplanted into the central nervous system. Nature 259: $567-570$.

Fonnum, F., R. Lund Karlsen, D. Malthe-Sørenssen, K. K. Skrede, and I. Walaas (1979) Localization of neurotransmitters, particularly glutamate, in hippocampus, septum, nucleus accumbens and superior colliculus. Prog. Brain Res. 51: 167191.

Gentschev, T., and C. Sotelo (1973) Degenerative patterns in the ventral cochlear nucleus of the rat after primary deafferentation. An ultrastructural study. Brain Res. 62: 37-60.

Gilad, G. M., and D. J. Reis (1979) Transneuronal effects of olfactory bulb removal on choline acetyltransferase and glutamic acid decarboxylase activities in the olfactory tubercle. Brain Res. 178: 185-190.

Goldowitz, D., S. R. Vincent, J. -Y. Wu, and T. Hökfelt (1982) Immunohistochemical demonstration of plasticity in GABA neurons of the adult rat dentate gyrus. Brain Res. 238: 413420 .

Graham, J., and V. A. Casagrande (1980) A light microscopic and electron microscopic study of the superficial layers of the superior colliculus of the tree shrew (Tupaia glis). J. Comp. Neurol. 191: 133-151.

Grofová, I., O. P. Ottersen, and E. Rinvik (1978) Mesencephalic and diencephalic afferents to the superior colliculus and periaqueductal gray substance demonstrated by retrograde axonal transport of horseradish peroxidase in the cat. Brain Res. 146: 205-220.

Hendrickson, A. E., M. P. Ogren, J. E. Vaughn, R. P. Barber, and J. -Y. Wu (1983) Light and electron microscopic immunocytochemical localization of glutamic acid decarboxylase in monkey geniculate complex: Evidence for GABAergic neurons and synapses. J. Neurosci. 3: 1245-1262.

Houser, C. R., and J. E. Vaughn (1981) Reorganization of GABAergic synapses in partially deafferented superior colliculus. Anat. Rec. 199: 120.

Houser, C. R., J. E. Vaughn, R. P. Barber, and F. Roherts (1980) GABA neurons are the major cell type of the nucleus reticularis thalami. Brain Res. 200: 341-354.

Kawamura, S., N. Fukushima, S. Hattori, and M. Kudo (1980) Laminar segregation of cells of origin of ascending projections from the superficial layers of the superior colliculus in the cat. Brain Res. 184: 486-490.

Kayama, Y., Y. Fukuda, and K. Iwama (1980) GABA sensitivity of neurons of the visual layer in the rat superior colliculus. Brain Res. 192: 121-131.

Lund, R. D. (1969) Synaptic patterns of the superficial layers of the superior colliculus of the rat. J. Comp. Neurol. 135 . 179-208.

Lund, R. D., and J. S. Lund (1971a) Modifications of synaptic 
patterns in the superior colliculus of the rat during development and following deafferentation. Vision Res. 11 (Suppl. 3): $281-298$.

Lund, R. D., and J. S. Lund (1971b) Synaptic adjustment after deafferentation of the superior colliculus of the rat. Science 171: 804-807.

McIlwain, J. T., and H. L. Fields (1971) Interactions of cortical and retinal projections on single neurons of the cat's superior colliculus. J. Neurophysiol. 34: 763-772.

McLaughlin, B. J., R. Barber, K. Saito, E. Roberts, and J. Y. $W_{1}$ (1975) Immunocytochemical localization of glutamate decarboxylase in rat spinal cord. J. Comp. Neurol. 164: 305322.

Mize, R. R., R. F. Spencer, and P. Sterling (1981) Neurons and glia in cat superior colliculus accumulate $\left[{ }^{3} \mathrm{H}\right]$ gamma-aminobutyric acid (GABA). J. Comp. Neurol. 202: 385-396.

Nadler, J. V. (1981) Desensitization-like changes in GABA receptor binding of rat fascia dentata after entorhinal lesion. Neurosci. Lett. 26: 275-281.

Nadler, J. V., C. W. Cotman, and G. S. Lynch (1974) Biochemical plasticity of short-axon interneurons: Increased glutamate decarboxylase activity in the denervated area of rat dentate gyrus following entorhinal lesions. Exp. Neurol. 45: $403 \sim 413$.

Nadler, J. V., W. F. White, K. W. Vaca, and C. W. Cotman (1977) Calcium-dependent $\gamma$-aminobutyrate release by interneurons of rat hippocampal regions: Lesion-induced plasticity. Brain Res. 131: 241-258.

Nieoullon, A., and N. Dusticier (1981) Increased glutamate decarboxylase activity in the red nucleus of the adult cat after cerebellar lesions. Brain Res. 224: 129-139.

Oertel, W. H., D. E. Schmechel, E. Mugnaini, M. L. Tappaz, and I. J. Kopin (1981) Immunocytochemical localization of glutamate decarboxylase in rat cerebellum with a new antiserum. Neuroscience 6: 2715-2735.

Ogren, M. P., A. E. Hendrickson, J. Vaughn, R. P. Barber, and J. Y. Wu (1982) GABAergic neurons and synapses in monkey dorsal lateral geniculate: A light and electron microscopic innunohistuchemical analysis. Soc. Neurosci. Abstr. 8: 262.

Ohara, P. T., A. R. Lieberman, S. P. Hunt, and J. -Y. Wu (1983) Neural elements containing glutamic acid decarboxylase (GAD) in the dorsal lateral geniculate nucleus of the rat: Immunohistochemical studies by light and electron microscopy. Neuroscience 8: 189-211.

Okada, Y. (1976) Distribution of GABA and GAD activity in the layers of superior colliculus of the rabbit. In $G A B A$ in Nervous System Function, E. Roberts, T. N. Chase, and D. B. Tower, eds., pp. 229-233, Raven Press, New York.

Rakic, P. (1975) Local circuit neurons. Neurosci. Res. Program Bull. 13: 333.

Ribak, C. E. (1978) Aspinous and sparsely-spinous stellate neurons in the visual cortex of rats contain glutamic acid decarboxylase. J. Neurocytol. 7: 461-478.

Ribak, C. E., J. E. Vaughn, K. Saito, R. Barber, and E. Roberts (1976) Immunocytochemical localization of glutamate decarboxylase in rat substantia nigra. Brain Res. 116: 287-298.

Ribak, C. E., J. E. Vaughn, and K. Saito (1978) Immunocytochemical localization of glutamic acid decarboxylase in neuronal somata following colchicine inhibition of axonal transport. Brain Res. 140: 315-332.

Ribak, C. E., J. E. Vaughn, and E. Roberts (1980) GABAergic nerve terminals decrease in the substantia nigra following hemitransections of the striatonigral and pallidonigral pathways. Brain Res. 192: 413-420.

Ribak, C. E., J. E. Vaughn, and R. P. Barber (1981) Immunocytochemical localization of GABAergic neurones at the electron microscopical level. Histochem. J. 13: 555-582.
Rustioni, A., and C. Sotelo (1974) Some effects of chronic deafferentation on the ultrastructure of the nucleus gracilis of the cat. Brain Res. 73: 527-533.

Saito, K., J. -Y. Wu, T. Matsuda, and E. Roberts (1974) Immunochemical comparisons of vertebrate glutamic acid decarboxylase. Brain Res. 65: 277-285.

Slemmon, J. R., P. M. Salvaterra, and K. Saito (1980) Preparation and characterization of peroxidase:antiperoxidase-Fab complex. J. Histochem. Cytochem. 28: 10-15.

Smith, L. M., and F. F. Ebner (1980) Synaptic response in a simple cortical system to thalamic removal: 'Type of synapse and postsynaptic targets of two different sprouting fiber systems. Soc. Neurosci. Abstr. 6: 328.

Sterling, P. (1971) Receptive fields and synaptic organization of the superficial gray layer of the cat superior colliculus. Vision Res. 11 (Suppl. 3): 309-328.

Sternberger, L. A. (1979) Immunocytochemistry, Ed. 2, pp. 104169, John Wiley \& Sons, Inc., New York.

Storm-Mathisen, J. (1976) Distribution of the components of the GABA system in neuronal tissue: Cerebellum and hippocampus-Effects of axotomy. In GABA in Nervous System Function, E. Roberts, T. N. Chase, and D. B. Tower, eds., pp. 149-68, Raven Press, New York.

Storm-Mathisen, J. (1977) Localization of transmitter candidates in the brain: The hippocampal formation as a model. Prog. Neurobiol. 8: 119-181.

Streit, P., E. Knecht, J. -C. Reubi, S. P. Hunt, and M. Cuénod (1978) GABA-specific presynaptic dendrites in pigeon optic tectum: A high resolution autoradiographic study. Brain Res. 149: 204-210.

Takahashi, Y., T. Ogawa, T. Takimori, and H. Kato (1977) Intracellular studies of rabbit's superior colliculus. Brain Res. 123: $170-175$.

Trigges, M., and J. Tigges (1975) Presynaptic dendrite cells and two other classes of neurons in the superficial layers of the superior colliculus of the chimpanzee. Cell Tissue Res. 162: 279-295.

Valverde, F. (1973) The neuropil in superficial layers of the superior colliculus of the mouse. A correlated Golgi and electron microscopic study. Z. Anat. Entwicklungsgesch. 142: 117-147.

Vaughn, J. E., R. P. Barber, C. E. Ribak, and C. R. Houser (1981a) Methods for the immunocytochemical localization of proteins and peptides involved in neurotransmission. In Current Trends in Morphological Techniques, J. E. Johnson, ed., Vol. 3, pp. 33-70, CRC Press, Inc., Boca Raton, FL.

Vaughn, J. E., E. V. Famiglietti, Jr., R. P. Barber, K. Saito, E. Roberts, and C. E. Ribak (1981b) GABAergic amacrine cells in rat retina: Immunocytochemical identification and synaptic connectivity. J. Comp. Neurol. 197: 113-127.

Vincent, S. R., T. Hattori, and E. G. McGeer (1978a) The nigrotectal projection: A biochemical and ultrastructural characterization. Brain Res. 151: 159-164.

Vincent, S. R., J. I. Nagy, and H. C. Fibiger (1978b) Increased striatal glutamate decarboxylase after lesions of the nigrostriatal pathway. Brain Res. 143: 168-173.

Westrum, L. E., and R. G. Black (1971) Fine structural aspects of the synaptic organization of the spinal trigeminal nucleus (pars interpolaris) of the cat. Brain Res. 25: 265-287.

Wood, J. G., B. J. McLaughlin, and J. E. Vaughn (1976) Immunocytochemical localization of GAD in electron microscopic preparations of rodent CNS. In GABA in Nervous System Function, E. Roberts, T. N. Chase, and D. B. Tower, eds., pp. 133-148, Raven Press, New York.

Wu, J. - Y., T. Matsuda, and E. Roberts (1973) Purification and characterization of glutamate decarboxylase from mouse brain. J. Biol. Chem. 248: 3029-3034. 